\title{
Thoracoscopic sympathetic clipping for hyperhidrosis: Long-term results and reversibility
}

\author{
Hiroshi Sugimura, MD, Ernest H. Spratt, MD, Christopher G. Compeau, MD, Deepa Kattail, and Yaron Shargall, MD
}

\begin{abstract}
Objective: The study objectives were to assess 1) postoperative satisfaction and the occurrence of compensatory sweating after endoscopic thoracic sympathetic clipping in a consecutive series of patients and 2) the reversibility of adverse effects by removing the surgical clips.
\end{abstract}

Methods: Between June 1998 and March 2006, 727 patients undergoing bilateral endoscopic thoracic sympathetic clipping for hyperhidrosis or facial blushing were prospectively followed for postoperative satisfaction and subjective compensatory sweating. The effect of removing the surgical clips was assessed in 34 patients who underwent a subsequent reversal procedure after endoscopic thoracic sympathetic clipping. Satisfaction and compensatory sweating were assessed using a visual analogue scale ranging from 0 to 10 , with 10 indicating the highest degree.

Results: Follow-up was complete in 666 patients (92\%). The median age was 26.9 years, and $383(53 \%)$ were men. The level of sympathetic clipping was T2 in 399 patients (55\%), T2+3 in 55 patients $(8 \%)$, and $\mathrm{T} 3+4$ in 273 patients (38\%). Median follow-up was 10.4 months (range 0-83 months). Excellent satisfaction ( $8-10$ on visual analogue scale) was seen at last follow-up in $288(74 \%)$ of the T2 group, $33(62 \%)$ of the T2+3 group, and $184(85 \%)$ of the T3+4 group. Postoperative satisfaction was significantly higher in the T3+4 group when compared with the T2 or T2+3 groups $(P<.01)$. Severe compensatory sweating $(8-10$ on the visual analogue scale) was reported in 42 $(13 \%)$ of the T2 group, $11(28 \%)$ of the T $2+3$ group, and $17(8 \%)$ of the T3+4 group. Postoperative compensatory sweating was significantly lower in the $\mathrm{T} 3+4$ group when compared with the $\mathrm{T} 2$ or $\mathrm{T} 2+3$ groups $(P<.05)$. Thirty-four patients have subsequently undergone removal of the surgical clips after endoscopic thoracic sympathetic clipping. Follow-up was complete in 31 patients. The reasons for removal included severe compensatory sweating in 32 patients, anhydrosis of the upper limb in 4 patients, lack of improvement or recurrence of hyperhidrosis in 5 patients, and other adverse symptoms in 5 patients. The reversal procedure was done after a median time of 11.0 months (range 157 months) after endoscopic thoracic sympathetic clipping. The initial level of clipping was T 2 in 21 patients, T2+3 in 7 patients, and T3+4 in 6 patients. There was a trend toward fewer subsequent reversal procedures in the T3+4 group when compared with the T2 or T $2+3$ groups $(P=.06)$. Fifteen patients $(48 \%)$ reported a substantial decrease in their compensatory sweating (5-10 on the visual analogue scale) after reversal. Thirteen patients $(42 \%)$ reported that their initial hyperhidrosis or facial blushing has remained well controlled ( $8-10$ on the visual analogue scale) after reversal. There was no significant relationship between the original level of clipping and the interval between endoscopic thoracic sympathetic clipping and the subsequent reversal and reversibility of symptoms.

Conclusion: When compared with endoscopic thoracic sympathetic clipping at the T2 or T2+3 levels, endoscopic thoracic sympathetic clipping at the T3+4 level was associated with a higher satisfaction rate, a lower rate of severe compensatory sweating, and a trend toward fewer subsequent reversal procedures. Subjective reversibility of adverse effects after endoscopic thoracic sympathetic clipping was seen in approximately half of the patients who underwent endoscopic removal of surgical clips. Although yet to be supported by electrophysiologic studies, reversal of sympathetic clipping seems to provide acceptable results and should be considered in selected patients.

Idiopathic or primary hyperhidrosis $(\mathrm{HH})$ is a chronic condition in which excessive sweating is seen in a focal pattern, usually affecting the palms, axillae, or soles. If symptoms

From the Division of Thoracic Surgery, University of Toronto, St Joseph's Health Centre, Toronto, Ontario, Canada.

Received for publication Aug 29, 2007; revisions received Dec 11, 2008; accepted for publication Jan 13, 2009.

Address for reprints: Yaron Shargall, MD, St Joseph's Health Centre, SSW-221, 30 The Queensway, Toronto, Ontario M6R 1B5, Canada (E-mail: shargy@stjoe.on. ca).

J Thorac Cardiovasc Surg 2009; 137:1370-8

$0022-5223 / \$ 36.00$

Copyright (C) 2009 by The American Association for Thoracic Surgery doi: $10.1016 /$ j.jtcvs. 2009.01 .008 are severe, $\mathrm{HH}$ can be socially and professionally disabling, and can significantly interfere with daily activities. Although $\mathrm{HH}$ is thought to be underreported, its prevalence has been reported as $2.8 \%$ in the United States ${ }^{1}$ and $4.9 \%$ in China. ${ }^{2}$ The cause of primary HH remains unknown.

When conservative measures in the treatment of $\mathrm{HH}$ fail, surgical treatment may be considered. First reported in 1920, sympathectomy has become an accepted method for treating $\mathrm{HH},{ }^{3}$ currently performed almost exclusively using videoassisted techniques. Although thoracoscopic interruption of the sympathetic trunk is associated with a high success rate and low morbidity when used in the treatment of $\mathrm{HH}$, compensatory sweating (CS) remains the most commonly 


\section{Abbreviations and Acronyms \\ $\mathrm{CS}=$ compensatory sweating \\ ETS $=$ endoscopic thoracic sympathetic clipping \\ $\mathrm{HH}=$ hyperhidrosis \\ VAS $=$ visual analogue scale}

reported side effect, with severe and debilitating symptoms reported in approximately $2 \%$ to $30 \%$ of patients. ${ }^{4-12}$ Various technical modifications have been attempted to achieve the highest patient satisfaction through optimal control of $\mathrm{HH}$, while avoiding the occurrence of significant CS. One such approach is the method of clamping the sympathetic nerve with surgical clips (endoscopic thoracic sympathetic clipping [ETS]), rather than cutting, coagulating, or partially removing the nerve. ${ }^{13}$ This method has the theoretic advantage of reversibility of adverse effects of sympathectomy by simple removal of the surgical clips if postsympathectomy side effects are severe. A few authors have reported mixed results for reversal in case reports or small case series. ${ }^{5,12-14}$ Yet, in the limited number of reported cases, the factors affecting reversibility remain largely unknown. The purpose of this study was therefore to assess the long-term satisfaction and significance of CS after ETS in a consecutive series of patients; to evaluate the effect of removal of surgical clips after ETS on satisfaction, CS, and $\mathrm{HH}$; and to define factors that might adversely or positively affect the outcome of the reversal procedure.

\section{MATERIALS AND METHODS}

Between June 1998 and March 2006, all patients undergoing sympathetic nerve block, sympathectomy, or sympathetic clipping for $\mathrm{HH}$ or facial blushing at St Joseph's Health Center in Toronto were included in a prospective follow-up study evaluating the results of their surgery. The degree of postoperative $\mathrm{HH}$ or facial blushing, as well as satisfaction rate (defined as relief of original symptoms and lack of side effects) and degree of CS after sympathetic surgery, were assessed at each follow-up event using a visual analogue scale (VAS) ranging from 0 to 10 , with 10 indicating the highest degree of each index (Appendix 1). The first follow-up was 1 to 4 weeks after surgery and then subsequently at 6- to 12-month intervals, through clinic visits or telephone/e-mail interviews.

Only patients undergoing bilateral ETS under our current standardized technique were included in the current analysis. All procedures were performed under general anesthesia with single-lumen endotracheal intubation. Patients were placed in a supine position with the arms abducted. Two 5-mm ports were introduced in the midaxillary line at the third and fifth intercostal spaces. We routinely used the Ternamian Endo TIP (Karl Storz, Tuttlingen, Germany) cannula for initial access into the pleural space. The pleural space was then insufflated with carbon dioxide to a pressure of less than $15 \mathrm{~mm}$ $\mathrm{Hg}$, and the second instrument port was introduced under direct vision. The parietal pleura overlying the sympathetic nerve was incised below the rib at the desired level of clipping (ie, below the second rib for T2 clipping and below the third and fourth ribs for $\mathrm{T} 3+4$ clipping), and the nerve was double-clipped using the 5-mm Endoclip applier (autosuture endoscopic clips). After clip application, $5 \mathrm{~mL}$ of $0.25 \%$ bupivacaine solution was injected along the site of pleural dissection for postoperative analgesia. The lung was reinflated under direct vision, and all port sites were closed primarily with absorbable stitches. Postoperative chest $\mathrm{X}$-ray was performed in the recovery unit, and the patient was discharged after 2 to 3 hours of monitoring in the postoperative unit.

The most recent follow-up information available at the time of analysis was used in the assessment of postoperative satisfaction and degree of CS after ETS. For patients who subsequently underwent a redo or reversal procedure, data collected immediately before the subsequent procedure were used in the analysis, as well as the most recent follow-up data after the second procedure.

To obtain procedure-specific data, all patients who underwent reversal (removal of surgical clip after ETS) were contacted via telephone or email and asked to complete a separate questionnaire in which they were asked to grade their satisfaction, degree of CS, and degree of $\mathrm{HH}$ after their reversal procedure (Appendix 2). The effect of the reversal procedure was analyzed while comparing the anatomic level of ETS, the primary indication for ETS, the time period since ETS, and the indication for reversal.

Data were presented as mean \pm standard deviation. Difference in satisfaction and degree of CS among groups was determined by the KruskalWallis test, and multiple comparison post-tests were performed by the Tukey-Kramer method. Proportional difference among groups was determined using the chi-square test. All statistical analyses were performed with SAS-based software (JMP version 5, SAS Institute, Cary, NC).

\section{RESULTS \\ Patients}

A total of 750 patients treated surgically for primary $\mathrm{HH}$ or facial blushing were identified, 727 of whom were the subject of this study. Twenty-three patients were excluded from the analysis because of alternative surgical techniques, including unilateral surgery, lumbar sympathectomy, open thoracotomy, or a combination of methods.

There were 383 male patients $(53 \%)$ and 344 female patients. The median age was 26.9 years (range, $14-65$ years), with 106 patients aged 10 to 20 years, 329 patients aged 21 to 30 years, 209 patients aged 31 to 40 years, and 83 patients aged more than 41 years. Among the 727 eligible patients, follow-up was complete in $666(92 \%)$. Median follow-up was 10.4 months (range, $0-83$ months).

The primary indication for surgery was palmar HH in 538 patients, facial blushing or craniofacial sweating in 173 patients, and axillary $\mathrm{HH}$ in 16 patients (Figure 1). The anatomic level of ETS was T2 (ETS-T2) in 399 patients, $\mathrm{T} 2+3($ ETS-T2+3) in 55 patients, and T3+4 (ETS-T3+4) in 273 patients (Figure 2). During the study period, our preferred surgical method has altered from the ETS-T2 level for all indications, including facial blushing, craniofacial, palmar, and axillary HH, to the ETS-T3+4 level for primarily palmar HH. This change was a result of growing evidence showing that interruption of the sympathetic trunk at the $\mathrm{T} 2$ level is unnecessary in the control of palmar $\mathrm{HH}$ and might be associated with a higher rate of severe CS. ${ }^{15-19}$ In recent years we have discouraged patients from having T2 clipping because of unsatisfactory results and higher incidence of severe CS. During the study period, 57 patients underwent more than 1 procedure. This included 25 $(3.4 \%)$ redo ETS procedures and $34(4.7 \%)$ reversals. 


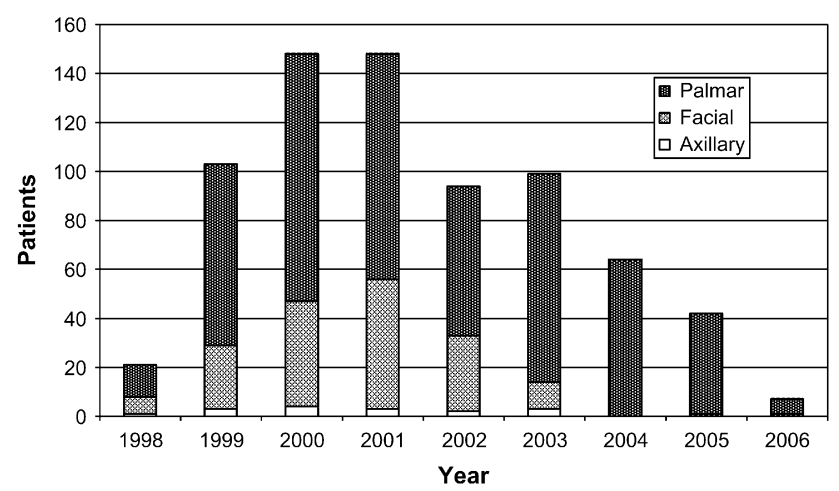

FIGURE 1. Surgical indication by year of surgery.

\section{Postoperative Results and Adverse Outcomes}

Surgical complications were rare. Chest tube insertion and drainage with subsequent hospital stay was necessary in 3 patients $(0.4 \%)$ with pneumothorax and 1 patient because of take-down of excessive adhesions. One patient required conversion to a thoracotomy because of major bleeding as a result of a superior vena cava tear. She recovered well after surgical repair and experienced good control of her HH. Horner's syndrome developed in 1 patient after ETS-T2+3, and reversal was requested after 3 months. One patient returned to the emergency department with a mild fever on postoperative day 2 but did not require treatment or hospital admission.

Postoperative results after ETS are shown in Table 1. Among 666 patients with follow-up, 508 (76\%) reported excellent satisfaction (8-10 on VAS). The mean satisfaction score for all patients was $8.2 \pm 2$. Excellent satisfaction was seen in $288(74 \%)$ of the T2 group, $33(62 \%)$ of the $\mathrm{T} 2+3$ group, and $184(85 \%)$ of the T3+4 group. Postoperative satisfaction was significantly higher in the T3+4 group when compared with the $\mathrm{T} 2$ or $\mathrm{T} 2+3$ groups $(P<.05)$.

The mean VAS score for the degree of persistent $\mathrm{HH} /$ facial blushing after ETS was $1.9 \pm 1.8$ for all patients. The mean score was $2.2 \pm 1.9$ for the $\mathrm{T} 2$ group, $1.6 \pm 1.7$ for the $\mathrm{T} 2+3$ group, and $1.5 \pm 1.3$ for the T3+4 groups, respectively. The degree of persistent $\mathrm{HH} /$ facial blushing was significantly less

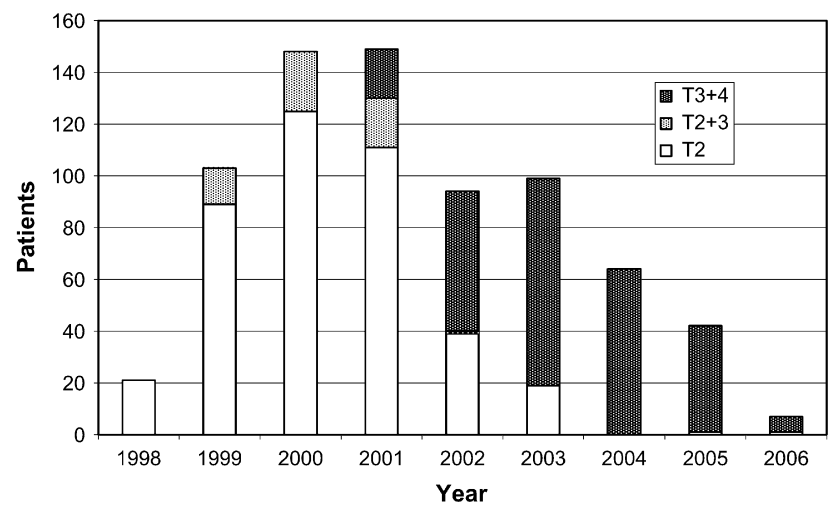

FIGURE 2. Level of sympathetic clipping by year of surgery.
TABLE 1. Postoperative results and adverse outcomes (visual analogue scale score)

\begin{tabular}{lcccc}
\hline Level of ETS & T2 & T2 $+\mathbf{3}$ & T3 +4 & \\
\hline $\mathrm{n}$ & 397 & 57 & 212 & \\
$\mathrm{HH} / \mathrm{FB}$ & $2.2 \pm 1.9 \dagger$ & $1.6 \pm 1.7$ & $1.5 \pm 1.3 \dagger$ & $P<.01^{*}$ \\
$\mathrm{CS}$ & $4.3 \pm 2.7 \dagger$ & $5.3 \pm 2.9 \ddagger$ & $3.7 \pm 2.5 \dagger, \ddagger$ & $P<.01^{*}$ \\
Satisfaction & $8.0 \pm 2.4 \dagger$ & $7.3 \pm 3.0 \ddagger$ & $8.8 \pm 1.8 \dagger, \ddagger$ & $P<.01^{*}$ \\
\hline ETS, Endoscopic thoracic sympathetic clipping; $C S$, compensatory sweating; $H H$, hy- \\
perhidrosis; $F B$, facial blushing. $* P<.01$ for comparison among all groups. $\dagger P<.05$ \\
for comparison between groups T2 and T3 $+4 . \ddagger P<.05$ for comparison between groups \\
T2 +3 and T3+4.
\end{tabular}

in the T3+4 group when compared with the T2 group $(P<$ $.05)$, but not when compared with the T2+3 group.

Severe CS (8-10 on VAS) was reported in $49(15 \%)$ of the T2 group, $13(24 \%)$ of the T2+3 group, and $17(8 \%)$ of the T3+4 group. The mean CS score was $4.3 \pm 2.7$ for the T2 group, $5.3 \pm 2.9$ for the $\mathrm{T} 2+3$ group, and $3.7 \pm 2.5$ for the T3+4 group. Postoperative CS was significantly lower in the $\mathrm{T} 3+4$ group when compared with the $\mathrm{T} 2$ or $\mathrm{T} 2+3$ groups $(P<.05)$. The proportion of patients reporting severe CS was significantly lower in the T3+4 group when compared with the combined $\mathrm{T} 2$ and $\mathrm{T} 2+3$ group $(P<.01)$.

When the primary indication for surgery was evaluated, 452 patients $(96 \%)$ treated for palmar HH, 138 patients $(89 \%)$ with facial blushing/sweating, and 15 patients $(94 \%)$ with axillary HH reported substantial relief from their primary symptoms ( $0-5$ on VAS) as a result of the ETS. Although $85 \%$ of patients with primary symptoms of palmar $\mathrm{HH}$ reported excellent satisfaction (8-10 on VAS), the rates of excellent satisfaction in patients with primary symptoms of facial blushing/sweating or axillary HH were only $62 \%$ and $53 \%$, respectively. However, the rates of moderate or excellent postoperative satisfaction (4-10 on VAS) were $98 \%, 92 \%$, and $87 \%$ for patients with palmar, facial, and axillary $\mathrm{HH}$, respectively (Figure 3 ). Thus, the majority of patients experienced at least moderate improvement of their symptoms after the surgical procedure.

\section{Redo Endoscopic Thoracic Sympathetic Clipping}

During the study period, 25 patients $(3.4 \%)$ underwent a redo ETS procedure. The level of initial ETS was T2 in 20

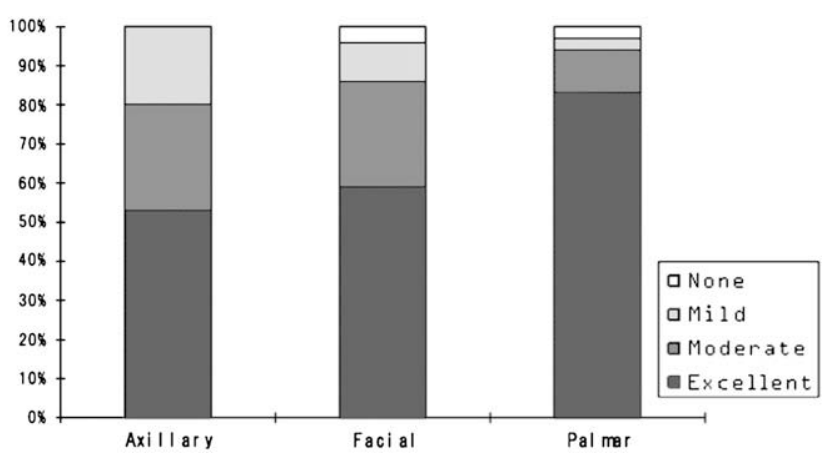

FIGURE 3. Postoperative satisfaction by surgical indication. 
patients, $\mathrm{T} 2+3$ in 1 patient, and $\mathrm{T} 3+4$ in 4 patients. The reason for redo ETS was suboptimal or unilateral control of symptoms in 20 patients and recurrent symptoms in 5 patients. The median interval between ETS and redo ETS was 3.5 months for suboptimal results and 21.1 months for recurrence. Sixteen of 18 patients underwent redo ETS after ETS-T2 because of suboptimal control of palmar HH. In 7 of these cases, ETS-T3+4 was performed at the time of redo surgery. In 1 case after ETS-T3+4, ETS-T2 was added to optimize control of facial $\mathrm{HH}$ and blushing. Seventeen patients $(74 \%$ of those with follow-up) reported excellent satisfaction after redo ETS.

\section{Reversals}

Thirty-four patients (4.7\%) subsequently underwent a reversal procedure with surgical removal of the clips after ETS. The procedure was done as a day procedure with a similar setup as for the original surgery. The previously placed incisions were used for port insertion. Mild adhesions were frequently observed along the clips. However, there were no or little adhesions between the lung and the chest wall. The overlying pleura was reincised, and the clips were removed by pulling the heel of the clip with a thin-nosed grasper. The sympathetic chain was kept intact. All cases were completed as planned, and there were no major complications or conversions to thoracotomy.

Among these patients, follow-up information after reversal was complete in $31(91 \%)$. Two patients were lost to follow-up, and 1 patient refused to participate in the study. The primary indication for reversal was severe CS in the majority $(\mathrm{n}=32)$ of patients. Other adverse symptoms seen in combination were anhydrosis of the upper limbs in 4 patients, lack of improvement or recurrence of $\mathrm{HH}$ in 5 patients, and Horner's syndrome and sexual dysfunction in 1 patient each.

The reversal procedure was done after a median time of 11.0 months (range, 0.6-56.9 months) after ETS. Median follow-up after the reversal procedure was 34.0 months (range, 0-72.1 months). The original level of the ETS was $\mathrm{T} 2$ in 21 patients $(5.3 \%$ of total $\mathrm{T} 2), \mathrm{T} 2+3$ in 8 patients $(14.5 \%$ of total $\mathrm{T} 2+3)$, and $\mathrm{T} 3+4$ in 5 patients $(1.8 \%$ of total $\mathrm{T} 3+4)$. There was a trend toward fewer subsequent reversal procedures in the $\mathrm{T} 3+4$ group when compared with the $\mathrm{T} 2$ and T $2+3$ groups $(P=.05)$. Table 2 summarizes postreversal results by the level of ETS. The mean time from ETS to reversal was $16.5 \pm 14.8$ months in the T2 group, $21.1 \pm 21.7$ months in the T2+3 group, and $11.7 \pm 17.6$ months in the $\mathrm{T} 3+4$ group. This was not significantly different among the groups. The follow-up period after reversal was significantly shorter in the $\mathrm{T} 3+4$ group when compared with the $\mathrm{T} 2$ group $(5.1 \pm 6.1$ months vs $34.0 \pm 20.3$ months, $P<.05)$.

Fifteen patients $(48 \%)$ reported a substantial decrease in their CS (5-10 on VAS) after reversal. Thirteen patients $(42 \%)$ reported that their initial HH or facial blushing has remained well controlled (8-10 on VAS) after reversal. There was no difference in the change in $\mathrm{CS}$ or the degree of $\mathrm{HH}$
TABLE 2. Reversal cases and postreversal results by level of endoscopic thoracic sympathetic clipping (visual analogue scale score)

\begin{tabular}{lcccc}
\hline \multicolumn{1}{c}{ Level of ETS } & T2 & T2 +3 & T3 +4 & \\
\hline $\mathrm{n}$ & 21 & 8 & 5 & \\
Time to reversal (mo) & $16.5 \pm 14.8$ & $21.1 \pm 21.7$ & $11.7 \pm 17.6$ & NS \\
Follow-up time & $34.0 \pm 20.3 \dagger$ & $30.4 \pm 27.0$ & $5.1 \pm 6.1 \dagger$ & $P<.05^{*}$ \\
$\quad$ after reversal (mo) & & & & \\
Change in CS & $4.5 \pm 3.4$ & $1.4 \pm 3.0$ & $3.2 \pm 2.9$ & NS \\
Control of HH & $5.5 \pm 3.9$ & $5.3 \pm 4.0$ & $6.8 \pm 4.3$ & NS \\
Satisfaction & $5.4 \pm 3.4 \ddagger$ & $1.3 \pm 1.9 \ddagger$ & $2.0 \pm 3.1$ & $P<.05^{*}$ \\
\hline
\end{tabular}

ETS, Endoscopic thoracic sympathetic clipping; $C S$, compensatory sweating; $H H$, hyperhidrosis; NS, not significant. ${ }^{*} P<.05$ for comparison among all groups. $\dagger P<.05$ for comparison between groups $\mathrm{T} 2$ and $\mathrm{T} 3+4 . \ddagger P<.05$ for comparison between groups $\mathrm{T} 2$ and $\mathrm{T} 2+3$.

after reversal among the T2, T2 +3 , and $\mathrm{T} 3+4$ groups $(P=$ .09 and $P=.72$ for universal comparison among all groups). Eight patients reported excellent satisfaction after reversal. Postreversal satisfaction was significantly better in the T2 group when compared with the $\mathrm{T} 2+3$ group (mean $5.4 \pm$ 3.4 vs $1.3 \pm 1.9, P<.05$ ). By using 6 months after ETS as the cutoff time period to compare "early" versus "late" reversal, 12 patients (of 34) received their reversal procedure within 6 months from their initial ETS procedure. The proportion of patients reporting substantial decrease in their CS after reversal was $67 \%$ in the early group and $37 \%$ in the late group ( $P=$ not significant). Postreversal satisfaction was excellent in $36 \%$ of the early group and $22 \%$ of the late group. Control of $\mathrm{HH}$ or facial blushing was well maintained in $25 \%$ and $53 \%$ of the early and late groups, respectively ( $P=$ not significant). Overall, there was no statistical difference in the postoperative results or satisfaction between the 2 groups (Table 3 ).

\section{DISCUSSION}

Primary or essential HH, defined as an excessive sweating beyond that expected physiologically, affects a significant proportion of the general population. Although benign in nature, it might cause significant social, emotional, and professional disabilities. There is a potential role for genetic predisposition, given a positive family history in up to $40 \%$ of the affected individuals. ${ }^{12}$ Social phobias are also common, mainly in combination with facial blushing and sweating, ${ }^{20,21}$ and add substantially to patients' discomfort.

Nonsurgical treatment methods, including topical absorbing powders, anticholinergic medications, botulinum toxin injections, and various biofeedback methods, are usually helpful only for patients with mild manifestations. Thus, the majority of patients with severe forms of $\mathrm{HH}$ will ultimately be offered a surgical sympathectomy as a definitive treatment.

Originally used for various other indications (eg, epilepsy, glaucoma, angina pectoris, reflex sympathetic dystrophy, and Raynaud's phenomenon), sympathectomy as a treatment for $\mathrm{HH}$ was first reported in $1920 .^{3}$ 
TABLE 3. Reversal cases and post-reversal results by time from initial endoscopic thoracic sympathetic clipping to reversal (visual analogue scale score)

\begin{tabular}{lccc}
\hline Time to reversal (mo) & $\leq \mathbf{6}$ mo & $>\mathbf{6}$ mo & \\
\hline $\mathrm{n}$ & 12 & 22 & \\
Change in CS & $4.5 \pm 3.4$ & $3.1 \pm 3.4$ & NS \\
Control of HH & $3.8 \pm 3.6$ & $5.4 \pm 4.1$ & NS \\
Satisfaction & $4.7 \pm 3.7$ & $3.3 \pm 3.4$ & NS \\
\hline
\end{tabular}

$\mathrm{CS}$, Compensatory sweating; $H H$, hyperhidrosis; $N S$, not significant.

At least 4 different open techniques were developed and widely used over the years. Those included the posterior, supraclavicular, anterior transthoracic, and axillary transthoracic approaches. ${ }^{22}$ Although generally effective in controlling the $\mathrm{HH}$, none of those techniques is simple, and the morbidity related to the open approach is substantial.

Endoscopic sympathectomy, developed over the past decade, has practically replaced the open techniques. Regardless of the method used to interrupt the sympathetic trunk, the overall results are satisfactory, the complication rate is small, and the morbidity is minimal.

There is little doubt that thoracoscopic sympathetic surgery has provided a highly reliable solution for palmar $\mathrm{HH}$. This is reflected in numerous clinical reports of successful control of symptoms by surgery in more than $95 \%$ of patients. $^{4,8-10,12,14,16}$ Our results are in keeping with those previous reports, with $96 \%$ of patients undergoing ETS for palmar $\mathrm{HH}$ reporting substantial improvement $(0-5$ on VAS) of their symptoms. This high success rate was associated with excellent satisfaction (8-10 on VAS) in $85 \%$ of our patients undergoing ETS for palmar $\mathrm{HH}$.

ETS was also highly successful in controlling the symptoms of facial blushing/sweating and axillary $\mathrm{HH}$ in our patients with success rates of 89 and $94 \%$, respectively. However, we should emphasize that successful control of symptoms was not necessarily associated with excellent satisfaction. In our series, despite the high success rates, the rate of excellent satisfaction was merely at the $50 \%$ to $60 \%$ range for these 2 groups of patients. Therefore, for axillary $\mathrm{HH}$ a local approach such as excision of sweat glands might be better in controlling HH symptoms.

The optimal surgical technique and level of sympathetic trunk interruption in thoracoscopic sympathetic surgery are still under debate. Direct comparison between studies is difficult because of the subjective characteristics and lack of uniformity in reporting the measured outcomes. Nevertheless, it is possible to draw some conclusions from the existing literature regarding ETS. First, the results are similar when comparing different techniques (clipping, ablation, cauterization, and division) but for the same indication (location of $\mathrm{HH}$ ) and at the same level of the sympathetic trunk. Thus, one should expect good results when operating on a patient with palmar $\mathrm{HH}$, regardless of the technique used.
Second, sympathectomy including the T2 level is associated with a success rate of approximately $90 \%$ to $100 \%$ in the treatment of craniofacial $\mathrm{HH}$ and facial blushing. However, it has been repeatedly reported that sympathectomy including the $\mathrm{T} 2$ level is associated with significantly worse CS and a lower degree of satisfaction postoperatively ${ }^{5,8,16,17,18}$ when compared with sympathectomy not including T2. Similarly in our series, ETS-T2 and ETS-T2+3 were both associated with a higher rate of severe CS and significantly lower postoperative satisfaction when compared with ETS-T3+4. There was also a trend toward more reversal procedures in the combined $\mathrm{T} 2$ and $\mathrm{T} 2+3$ groups when compared with the $\mathrm{T} 3+4$ group.

CS is the most significant adverse effect after sympathectomy. Approximately $10 \%$ of our patients sustained severe CS, more than the $6 \%$ reported by Reisfeld and colleagues ${ }^{12}$ in their large cohort (1312) of patients, but less than reported by other groups, who described up to $30 \%$ of patients with severe CS symptoms. ${ }^{7,9,18}$ The common hypothesis for CS is that this phenomenon is a thermoregulatory mechanism by which the sweat glands attempt to compensate for the decreased amount of secretory tissue. Several authors have therefore suggested that reducing the extent of sympathetic nerve interruption will result in reducing the occurrence of CS. ${ }^{17}$ Currently, however, there are no electrophysiologic studies to support these concepts. In addition, it has been suggested that the anatomic variations in the sympathetic trunk, including aberrant pathways between the upper thoracic and the intercostal nerves, enable fibers to bypass the sympathetic chain and might be one of the main reasons for failure of surgical sympathectomy. ${ }^{23}$ As a result, some surgeons are routinely dividing or coagulating all the aberrant fibers around the level of the sympathectomized chain. Yet, there are no studies to support this hypothesis, and no clinical data are available to support the routine use of this maneuver during ETS. In our experience, only $3.4 \%$ of the patients required redo surgery because of failure of the original procedure, and most of them simply required the addition of T3T4 clipping to a preexisting T2.

The main limitation in evaluating the results of surgical sympathectomy is the inability to objectively measure the outcome. Although most patients are satisfied with the results, all the methods for outcome measurement are of a subjective nature. Many centers have developed different scales/parameters to evaluate success rate after sympathectomy ETS, none of which is widely used by others. Only a few groups are routinely monitoring physiologic parameters, such as heart rate and skin temperature, during or after the surgical procedure. ${ }^{25}$ However, these parameters might not be precise or long-lasting.

Yet, in a procedure focused primarily on achieving high patient satisfaction, the main outcome is, indeed, the patient's subjective evaluation of his or her symptoms. Thus, it seemed reasonable to assess our patients using the VAS, 
which is easy to use and allows for a relatively simple and accurate self-assessment by patients.

The concept of potential reversibility of adverse effects after CS by clip removal is attractive and intuitively sound. After sympathectomy using nerve cutting or various other methods of nerve injury, the only reliable way to restore trunk continuity is by using a nerve graft to interpose between the cut/injured ends. ${ }^{24}$ This is a complex surgery that requires harvesting and anastomosis of nerves. However, if restoration of continuity in the sympathetic chain by removal of clips that initially crushed the chain will allow for renewal of conductivity in the trunk, then the method of sympathetic clipping (in which the continuity of the sympathetic trunk is maintained) is clearly preferable as the initial surgical treatment for $\mathrm{HH}$, because it maintains the option of effective and relatively simple reversibility. Before the current study, there were only a few reports assessing reversal of sympathetic clipping, ${ }^{5,12-14,16}$ most of which are case reports or small case series. Our group of 34 patients with reversal is the largest reported series to date. We are also the only group to follow their reversal patients prospectively for a relatively long period of time (up to 5 years). Although we realize that the T3T4 reversal group was followed for relatively short period (median of 5.1 months), simply reflecting the fact that we started performing T3T4 almost exclusively during the last 3 years, the follow-up for the other reversal groups was significantly longer. Thus, the substantial decrease in CS in approximately half of the patients after reversal is reliable, and the results were maintained in some patients for several years. We do not have a good explanation for the fact that approximately $40 \%$ of patients did not experience recurrence of their original sweating/blushing pattern after the reversal, especially because some did show reversal of their CS. It is unclear why a patient may experience reduction of CS after reversal while not experiencing recurrence of his/her original symptoms. Currently, there is no valid or reproductive animal model to clarify the actual mechanism of $\mathrm{HH}$ and to validate the concept of sympathectomy and reversal. The only reported in vivo animal model to evaluate the activity of the sympathetic chain within this concept was reported by Lavian and colleagues, ${ }^{26}$ who developed an electrode device that was implanted on the stellate ganglia of dogs to allow for a chronic animal model with continuous recording of neuroelectrical signals. They demonstrated long-term reproductive signal recording by the device but have not repeated it in the setup of a sympathectomy model. Thus, more research is clearly needed to support the concept of sympathectomy, and in particular that of reversibility.

\section{CONCLUSIONS}

What are the clinical implications of our study? First, the study provides additional evidence to support previous reports showing an excellent safety profile and long-term out- come for sympathectomy. Second, the study provides some evidence for the usefulness of reversibility. It seems that reversal might help a substantial proportion of patients with severe adverse effects after sympathectomy, and that the results of the reversal procedure are long-lasting. Third, the fact that approximately half of the patients undergoing reversal in our experience reported a substantial decrease in their $\mathrm{CS}$, but that approximately one third did not experience a recurrence of their original $\mathrm{HH}$ even after clip removal, cannot be simply explained by nerve regeneration after clip removal. Thus, some exciting questions are raised with regard to possible alternative mechanisms and the need for better understanding of the activity of the sympathetic chain, as well as its relationship to $\mathrm{HH}$.

\section{References}

1. Strutton DR, Kowalski JW, Glaser DA, Stang PE. US prevalence of hyperhidrosis and impact on individuals with axillary hyperhidrosis: results from a national survey. J Am Acad Dermatol. 2004;51:241-8.

2. Tu YR, Li X, Lin M, Lai FC, Li YP, Chen JF, et al. Epidemiological survey of primary palmar hyperhidrosis in Fuzhou of People's Republic of China. Eur J Cardiothorac Surg. 2007;31:737-9.

3. Hashmonai M, Kopelman D. History of sympathetic surgery. Clin Auton Res. 2003;13:6-9.

4. Neumayer C, Zacher I, Holak G, Fugger R, Jakesz R, Herbst F, et al. Limited endoscopic thoracic sympathetic block for heperhidrosis of the upper limb. Reduction of compensatory sweating by clipping T4. Surg Endosc. 2004;18:152-6.

5. Neumayer C, Panhofer P, Zacherl J, Bischof G. Effect of endoscopic thoracic sympathetic block on plantar hyperhidrosis. Arch Surg. 2005;140:676-80.

6. Kumagai K, Kawase H, Kawanishi M. Health-related quality of life after thoracoscopic sympathectomy for palmar hyperhidrosis. Ann Thorac Surg. 2005;80:461-6.

7. Dumont P, Denoyer A, Robin P. Long-term results of thoracoscopic sympathectomy for hyperhidrosis. Ann Thorac Surg. 2004;78:1801-7.

8. Schmidt J, Georges Bechara F, Altmeyer P, Zirngibl H. Endoscopic thoracic sympathectomy for severe hyperhidrosis: impact of restrictive denervation on compensatory sweating. Ann Thorac Surg. 2006;81:1048-55.

9. Doolabh N, Horswell S, Williams M, Huber L, Prince S, Meyer DM, et al. Thoracoscopic sympathectomy for hyperhidrosis: indications and results. Ann Thorac Surg. 2004;77:410-4.

10. Gossot D, Galetta D, Pascal A, Debrosse D, Caliandro R, Girard P, et al. Long term results of endoscopic thoracic sympathectomy for upper limb hyperhidrosis. Ann Thorac Surg. 2003;75:1075-9.

11. Reisfeld R, Nguyen R, Pnini A. Endoscopic thoracic sympathectomy for treatment of essential hyperhidrosis syndrome: Experience with 650 patients. Surg Laparosc Endosc Percutan Tech. 2000;10:5-10.

12. Reisfeld R, Nguyen R, Pnini A. Endoscopic thoracic sympathectomy for hyperhidrosis. Experience with both cauterization and clamping methods. Surg Laparosc Endosc Percutan Tech. 2002;12:255-67.

13. Lin C-C, Mo L-R, Lee L-S, Ng S-M, Hwang M-H. Thoracoscopic T2-sympathetic block by clipping-A better and reversible operation for treatment of hyperhidrosis palmaris: experience with 326 cases. Eur J Surg. 1998;580:13-6.

14. Lin T-S, Chou M-C. Treatment of palmar hyperhidrosis using needlescopic T2 sympathetic block by clipping: analysis of 102 cases. Int Surg. 2004;89:198-201.

15. Lin CC, Telaranta T. Lin-Telaranta classification: the importance of different procedures for different indications in sympathetic surgery. Ann Chir Gynaecol. 2001;90:161-6.

16. Reisfeld R. Sympathectomy for hyperhidrosis: should we place the clamps at T2 T3 or T3-T4? Clin Auton Res. 2006;16:384-9.

17. Dewey TM, Herbert MA, Hill SL, Prince SL, Mack MJ. One-year follow-up after thoracoscopic sympathectomy for hyperhidrosis: outcomes and consequences. Ann Thorac Surg. 2006;81:1227-33.

18. Chou S-H, Kao E-L, Lin C-C, Chang Y-T, Huang M-F. The importance of classification in sympathetic surgery and a proposed mechanism for compensatory hyperhidrosis: experience with 464 cases. Surg Endosc. 2006;20:1749-53.

19. Licht PB, Jorgensen DJ, Ladegaard L, Pilegaard HK. Thoracoscopic sympathectomy for axillary hyperhidrosis: the influence of T4. Ann Thorac Surg. 2005;80:455-60. 
20. Licht PB, Ladegaard L, Pilegaard HK. Thoracoscopic sympathectomy for isolated facial blushing. Ann Thorac Surg. 2006;81:1863-6.

21. Biebl MO, Fraedrich G, Larcher P, Biedermann H. Amelioration of specific phobias after thoracic sympathetic block in patients with localized hyperhidrosis. Ann Thorac Surg. 2007;83:1578-9.

22. Kopelman D, Hashmonai M. Upper thoracic sympathetic surgery: open surgical techniques. Clin Auton Res. 2003;13:10-5.

23. Schiller Y. The anatomy and physiology of the sympathetic innervation to the upper limbs. Clin Auton Res. 2003;13:1-5.

24. Teleranta T. Reversal surgery for reducing the side effects of ETS. A case report. Ann Chir Gyn. 2001;90:175-6.

25. Schick CH, Fronek K, Held A, Birklein F, Hohenberger W, Schmelz M. Differential effects of surgical sympathetic block on sudomotor and vasoconstrictor function. Neurology. 2003;60:1770-6.

26. Lavian G, Kopelman D, Shenhav A, Konyukhov E, Gardi U, Zaretzky A, et al. In vivo extracellular recording of sympathetic ganglion activity in a chronic animal model. Clin Auton Res. 2003;13:83-8.

\section{Discussion}

Dr James Allan (Boston, Mass). Thanks very much. That was very nicely presented and I want to congratulate you on adding a large body of data to the literature.

I find the data provocative in several respects. First, in your series, 25 patients $(3.4 \%)$ underwent repeat surgery due to a lack of effectiveness of the initial operation. The majority of these patients had initially been clipped at just the T2 level for palmar hyperhidrosis. In my personal experience of about 60 patients, I have never needed to reoperate on a patient; however, I perform a T2 to T4 ablation with division of accessory nerves, not just a T2 clipping. So I would like to know whether the initial failure in these patients reflects (1) that clipping at a given level may be inferior in efficacy to cutting or ablating at the same level, (2) that some patients simply require longer sympathectomies than others in an unpredictable fashion, or (3) that the initial failures are a reflection of performing a procedure that does not address accessory sympathetic fibers?

Dr Sugimura. Dr Allan, thank you very much for your comments and questions.

It is true that we had 25 redo surgeries that required redo because of suboptimal results or what looked like early recurrence of their symptoms, and the majority were in patients that received T2 clipping for palmar hyperhidrosis.

In regards to the difference between the clipping method and the cutting or ablation method, I think that our results using the clipping method are comparative to the results utilizing other methods in terms of satisfaction or treatment success. Some authors with experience with both methods even suggest that clipping was more effective. I do not think that the redos were a result of inferiority of the clipping method. I personally think that accessory sympathetic conduction might have been responsible, or at least in part, for suboptimal results or early recurrences resulting in redo. We do not routinely clip aberrant nerve fibers, and the fact that you see more aberrant fibers at the T1/T2 levels, as compared to the T3/T4 levels is the reason for my suspicion. Therefore when we perform redo sympathetic clipping, if we find an aberrant fiber at that time, we ablate that nerve. After experience with clipping at both T2 and T3/T4 levels, we now strongly believe that T3/T4 clipping is a far more superior method to $\mathrm{T} 2$ clipping that does not require further intervention to the accessory nerves. Because most of the redos were done following $\mathrm{T} 2$ clipping, in fact we have added clips to the T3/T4 levels in most of the cases at the time of redo.
Dr Allan. My second question for you relates to the 2 patients (among the 34 patients who underwent reversal) that did so for reasons other than compensatory hyperhidrosis. I believe from your manuscript, one of them may have had a Horner's syndrome and the other may have had sexual dysfunction, which is a complication that I was not aware of. Can you describe these 2 patients, if I've understood the presentation correctly, and what their outcome was after reversal?

Dr. Sugimura. The patient who presented with Horner's-like symptoms was a T2 clipping case done in the early era. It wasn't the typical presentation of symptoms, but there was a mild degree of ptosis and some bloodshot conjunctiva, which was among multiple complaints including significant compensatory sweating. We did perform reversal in that case.

Dr Allan. Was there symptomatic improvement in that patient after reversal?

Dr Sugimura. There was mild symptomatic relief in that patient. The patient presenting with sexual dysfunction also had an L2 to 4 chemical block of the sympathetic chain for plantar hyperhidrosis close to the time of thoracic sympathetic clipping.

Dr Allan. Finally, among the 32 patients who underwent reversal for compensatory hyperhidrosis, did you notice a correlation between the resolution of the compensatory hyperhidrosis and the subsequent recurrence of the primary symptom?

Dr Sugimura. As we showed in the presentation, there was a trend toward association between the two changes in the symptoms, meaning if you do an early reversal, you'll have more patients reporting significant relief from their compensatory sweating and at the same time less patients who can maintain their level of control of hyperhidrosis.

\section{Dr Allan. Thank you}

Dr Dan Meyer (Dallas, Tex). That was an excellent presentation.

I have three quick questions. Do you do any intraoperative monitoring, such as palmar temperature monitoring, to make sure that you have interrupted the blood supply during your sympathectomy?

Dr Sugimura. No, we do not.

Dr Meyer. Are there a group of patients that you've found that you've predicted preoperative factors that may predict severe compensatory sweating that you would not offer a sympathectomy to? Because there are patients who have diffuse sweating, worse in the hands or axillae, that you worry that they may get severe compensatory sweating postoperatively.

Dr Sugimura. I think it's more about the indication for clipping. If you are to treat a patient with a primary complaint of craniofacial hyperhidrosis, then we believe that it's more effective to perform a T2 clipping. However, we know that T2 clipping is associated with worse compensatory sweating. So if you call that a high-risk group for significant compensatory sweating, then that might be the case.

Dr Meyer. And the last question, have you considered just doing a T3 sympathectomy instead of the T3/T4 clipping? What do you think the gain of the addition of the T4 is?

Dr Sugimura. First of all, we don't have experience with singlelevel clipping. We are aware of reports showing excellent results and literally zero compensatory sweating with the single level sympathectomy at the lower level of the sympathetic trunk, and I agree that that is a method that we should look into in the future. I believe that a lot of people who are experienced in this surgery think that 
the less manipulation you do to the sympathetic trunk, the less adverse effects you create, and if you can achieve the same results with less manipulation, I think that is the optimal way to treat.

Dr Douglas Wood (Seattle, Wash). I want to follow up on Dr Allan's last question, which I guess I'm not sure we answered adequately. I think that he is asking whether in individual patients was there a correlation in time of when the sympathectomy was reversed and the recurrence of hyperhidrosis and relief of compensatory sweating? In individual patients was the relief of compensatory sweating accompanied by a recurrence of symptoms?

Dr Sugimura. It usually was. But there was a small number of patients who experienced relief from their compensatory sweating after reversal while enjoying persistent control of hyperhidrosis, and those patients were more in the T2 group-well, we had more reversals in the $\mathrm{T} 2$ group to start with. My personal feeling is that with all the measures that we use to look at the outcomes being so subjective, we have to be very careful in our attempt to quantify the relationship between these 2 outcomes. In some people with a huge burden of compensatory sweating, with even mild relief after reversal they might feel quite satisfied, regardless of the change in their state of hyperhidrosis. It's hard to really understand the quantitative association unless we do some confirmation with electrophysiological studies.

Dr Allan. I agree that patient selection is a key component to subsequent patient satisfaction. I would not want to bring $10 \%$ of my patients back to the operating room due to a failure to control the primary condition, or due to my creating a side effect that turns out to be worse than the primary condition. Therefore, I make a strong effort to be certain that the patient is significantly bothered by palmar hyperhidrosis. I do not typically offer surgery for isolated craniofacial hyperhidrosis, and also try to limit operating on isolated axillary symptoms, as the reported efficacy of sympathectomy for axillary symptoms is only about $70 \%$.

Dr Sugimura. Thank you very much. I couldn't agree more with that comment. We are being more and more selective in our patient selection. There are about $10 \%$ of patients who come to see us with complaints of solely craniofacial hyperhidrosis or axillary hyperhidrosis. Because we now know that sympathetic surgery in this population is more likely to fail or cause significant adverse effects, we basically discourage these patients from surgery.

\section{Appendix 1. Endoscopic thoracic sympathetic clipping follow-up questionnaire}

1. How would you rate your current degree of facial / hand / armpit / foot sweating, or facial blushing?

None

Debilitating

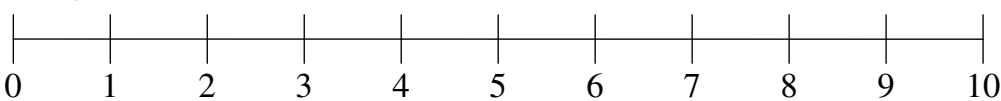

2. How would you rate your current degree of compensatory / reflex sweating?

None

Debilitating

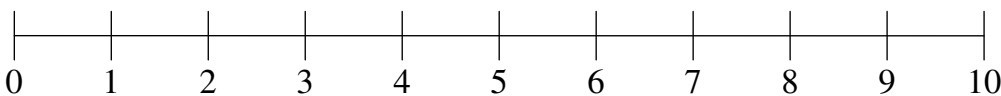

3. What is your current degree of satisfaction with the surgery (ETS)?

Not satisfied at all Completely satisfied

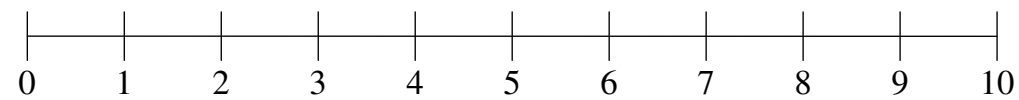

1. Location of compensatory sweating: - Face

(Mark all that apply) - Chest

- Abdomen

- Feet

- Neck

- Back

- Buttocks/thighs

- Gustatory

- None

2. Other adverse effects after sympathetic clipping: 


\section{Appendix 2. Endoscopic thoracic sympathetic clipping follow-up questionnaire (after reversal)}

1. How would you rate your current degree of facial / hand / armpit / foot sweating, or facial blushing?

Same as before ETS

Complete dryness

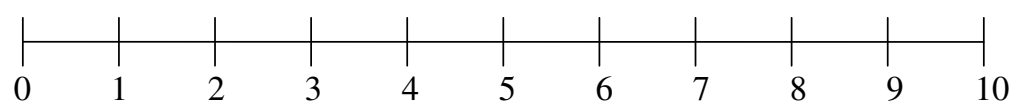

2. How would you rate your current degree of compensatory / reflex sweating?

Same as before reversal

Back to normal (before ETS)

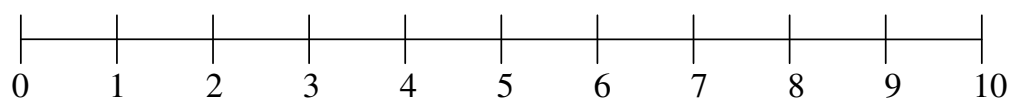

3. What is your current degree of satisfaction after reversal?

Not satisfied at all

Completely satisfied

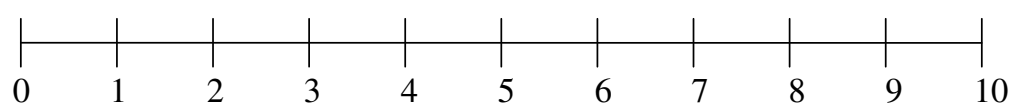

Other effects following removal of sympathetic clips: 